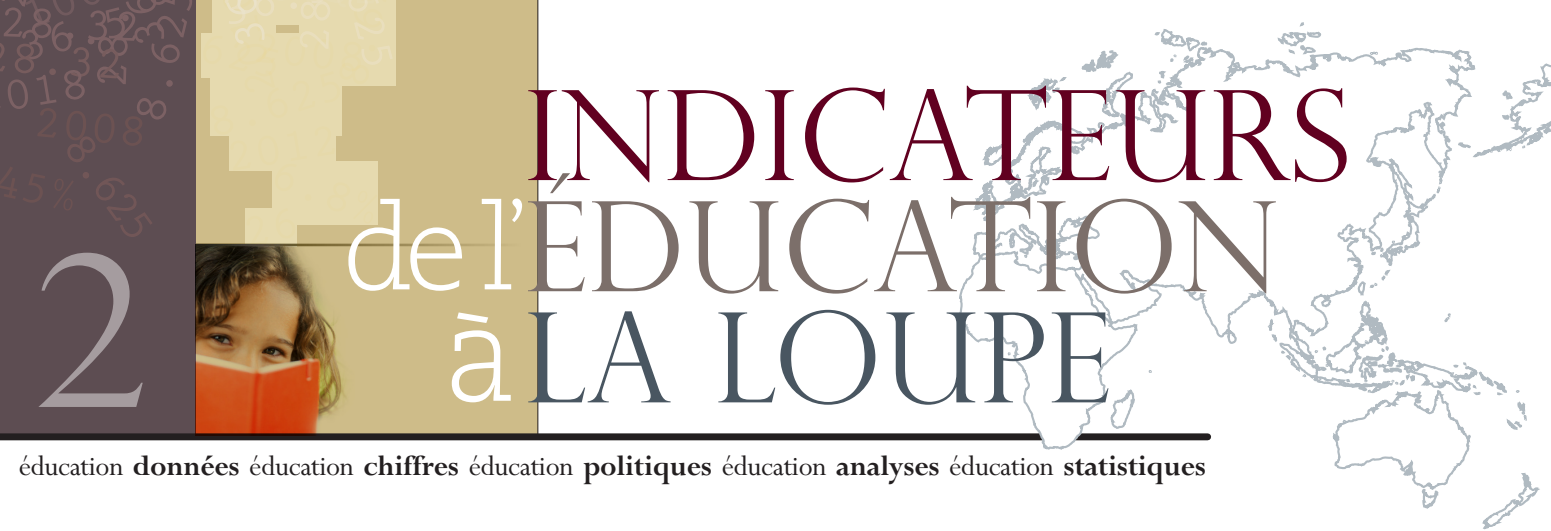

\title{
Quelles aides les pays apportent-ils aux étudiants de l'enseignement supérieur dans le monde?
}

- Les pays de l'OCDE où les étudiants bénéficient d'aides financières importantes affichent un taux d'accès à l'enseignement universitaire supérieur à la moyenne, même lorsque les frais de scolarité y sont comparativement élevés.

- Les systèmes d'aide financière qui combinent des prêts avec remboursement subordonné au revenu pour l'ensemble des étudiants avec des allocations sous condition de ressources peuvent être un moyen efficace de promouvoir l'accès à l'enseignement supérieur et son équité, tout en partageant le coût de ce dernier entre les pouvoirs publics et les étudiants.

- Dans un nombre croissant de pays de l'OCDE, les frais de scolarité sont plus élevés pour les étudiants en mobilité internationale, et dans de nombreux pays, ils varient également en fonction du domaine d'études.

Les systèmes de frais de scolarité et d'aide aux étudiants varient fortement entre les pays de l'OCDE.

En matière d'enseignement supérieur (ou tertiaire), de nombreux pays affichent des objectifs similaires : renforcer l'économie de la connaissance, augmenter les taux d'accès des étudiants, améliorer les taux de réussite et garantir la stabilité financière de leurs systèmes d'enseignement supérieur. Toutefois, il existe des différences fondamentales entre les pays de I'OCDE, tant en termes de répartition du coût de l'enseignement supérieur que de type d'aide financière proposée aux étudiants.

\section{Rapport entre les frais de scolarité moyens et la proportion d'étudiants bénéficiaires d'aides publiques au titre de l'enseignement supérieur, 2008-09}

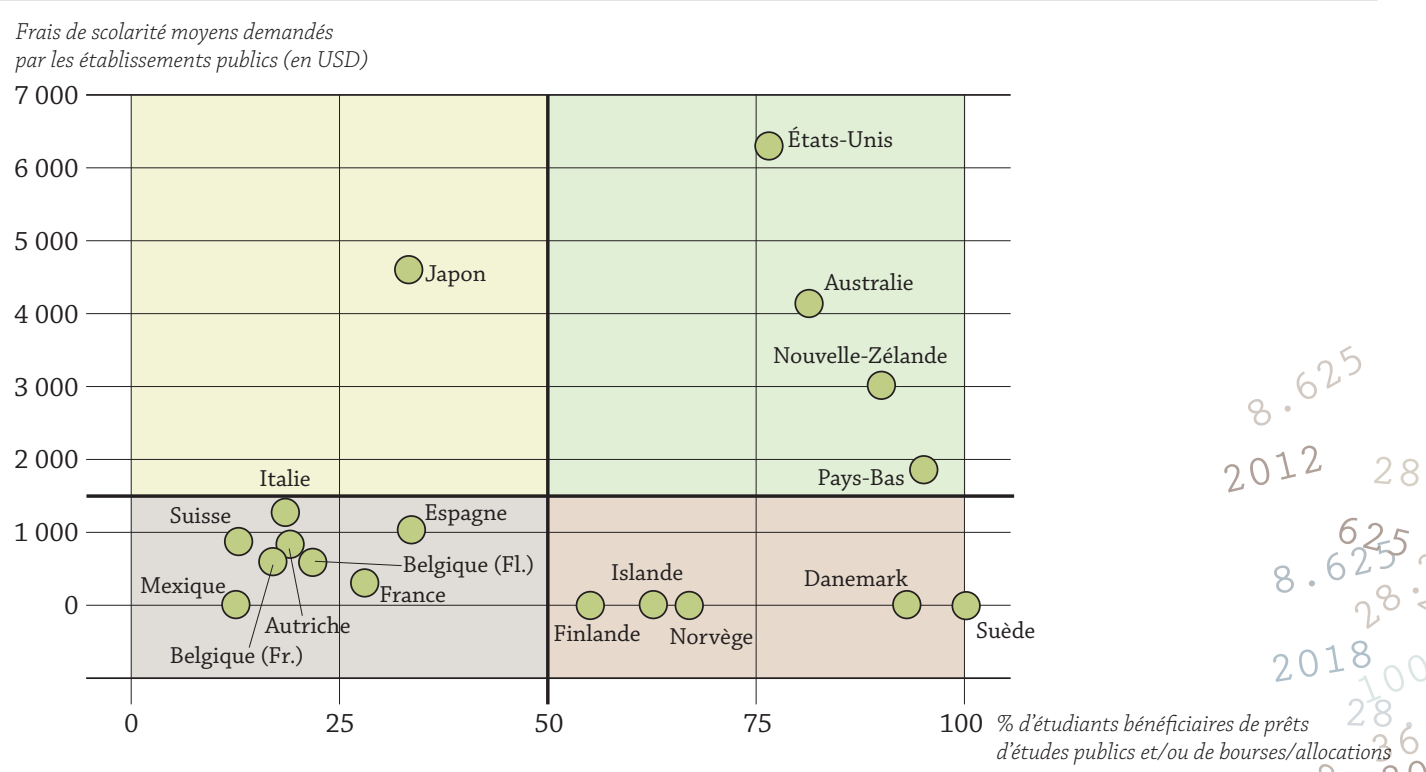

Remarque : ce graphique exclut les pays de l'OCDE dont les données spécifiquement relatives aux aides publiques ne sont pas disponibles. Source : Regards sur l'éducation 2011 : Les indicateurs de l'OCDE, Indicateur B5 (www.oecd.org/edu/eag2011). 
Ainsi, dans des pays offrant des structures fiscales plus progressives, tels que le Danemark, la Finlande, I'Islande, la Norvège et la Suède, les étudiants paient des frais de scolarité peu élevés, voire nuls, et bénéficient d'aides publiques généreuses au titre de l'enseignement supérieur, mais sont soumis à un taux élevé d'imposition sur le revenu. Par contraste, les frais de scolarité peuvent être nettement plus élevés en Australie, au Canada, aux États-Unis, en Nouvelle-Zélande et aux Pays-Bas, même si les étudiants de ces pays bénéficient également d'importantes aides financières. Avant de récentes réformes en Corée et au Japon, les étudiants devaient payer des frais de scolarité comparativement élevés, mais avaient un accès relativement limité aux aides publiques. Parallèlement, en Autriche, en Belgique, en Espagne, en France, en Irlande, en Italie, au Mexique, au Portugal, en République tchèque et en Suisse, les étudiants s'acquittent de frais de scolarité peu élevés, voire nuls, au titre de l'enseignement supérieur, mais ont un accès limité aux aides financières.

À l'heure où la plupart des pays de l'OCDE sont confrontés au double défi de l'essor des effectifs scolarisés dans l'enseignement supérieur et des réductions budgétaires, dans quelle mesure ces différentes approches permettent-elles aux systèmes d'enseignement supérieur des pays d'atteindre leurs principaux objectifs ?

\section{Dans de nombreux pays, un système bien développé d'aide financière aux étudiants joue un rôle primordial...}

Selon les études de l'OCDE, I'application de frais de scolarité modérés, accompagnée de la possibilité pour les étudiants de bénéficier de systèmes inclusifs d'aide financière, constitue un moyen efficace pour les pays d'accroître l'accès à l'enseignement supérieur, d'optimiser les ressources de budgets publics limités et de reconnaître l'importance du rendement privé d'une formation dans l'enseignement supérieur pour les étudiants. S'il n'est pas facile de définir ce que l'on entend par frais de scolarité "modérés », dans les pays de I'OCDE où l'enseignement supérieur est payant, le montant des frais de scolarité annuels va le plus souvent de 800 USD à 1300 USD par ressortissant national scolarisé à temps plein dans un programme universitaire. Dans chaque pays, un large éventail de facteurs influencent les taux d'accès à l'enseignement supérieur, notamment la qualité des systèmes d'enseignement primaire et secondaire, la prévalence des formations professionnelles et le nombre d'étudiants en mobilité internationale scolarisés dans l'enseignement supérieur.

Taux d'accès à l'enseignement universitaire

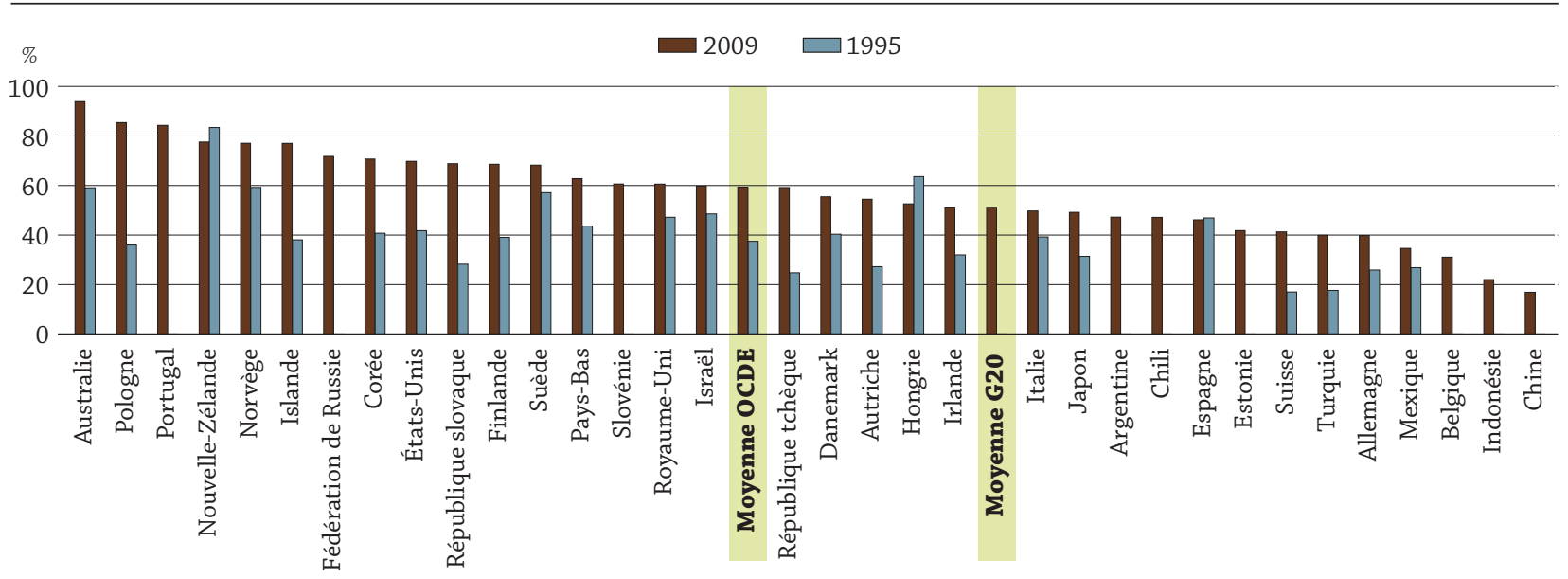

Ce graphique propose une estimation actuelle de la proportion de jeunes qui entameront une formation universitaire au cours de leur vie.

Remarque : pour les États-Unis, les taux d’accès de 2009 incluent également des étudiants qui ne sont pas scolarisés dans des formations universitaires. Source : Regards sur l'éducation 2011 : Les indicateurs de l'OCDE, Indicateur C2 (www.oecd.org/edu/eag2011). 
Il est toutefois intéressant de noter que des pays offrant des systèmes d'aide financière particulièrement bien développés - tels que l'Australie, les États-Unis, la Nouvelle-Zélande et le Royaume-Uni - présentent tous des taux d'accès à l'enseignement universitaire supérieurs à la moyenne de l'OCDE, bien qu'ils pratiquent des frais de scolarités très élevés. Néanmoins, force est de constater que cette approche a ses propres limites : si le coût de l'enseignement supérieur est perçu comme trop élevé, les individus sont susceptibles d'y renoncer, même s'ils peuvent bénéficier d'aides publiques pour le financer.

En outre, le niveau élevé des taux d'accès observé dans certains pays qui ne facturent pas de frais de scolarité peut également s'expliquer par la mise à disposition de leurs étudiants de systèmes très développés d'aide financière pour couvrir leurs frais de subsistance, et pas uniquement par l'absence de frais de scolarité. Ainsi, en Finlande, en Islande, en Norvège et en Suède - quatre pays où le taux d'accès à l'enseignement universitaire est supérieur à la moyenne - plus de $55 \%$ des étudiants bénéficient d'allocations, de prêts d'études publics, ou d'une combinaison des deux. Par contraste, les pays ne facturant pas de frais de scolarité, mais offrant des systèmes d'aide aux étudiants moins développés - tels que l'Irlande et le Mexique - accusent des taux d'accès moins élevés.

\section{... mais le type d'aide accordé aux étudiants est peut-être encore plus important.}

Parallèlement, l'existence d'un système solide d'aide financière peut ne pas s'avérer suffisante pour garantir la réussite des étudiants dans l'enseignement supérieur ; le type d'aide proposé joue également un rôle essentiel. Là encore, les approches varient selon les pays de I'OCDE : plus d'un tiers d'entre eux proposent des systèmes d'aide aux étudiants qui reposent exclusivement sur l'octroi d'allocations, de bourses ou de paiements directs aux universités. L'Islande ne propose que des prêts d'études, tandis que le reste des pays offrent une combinaison d'allocations et de prêts.

Aides publiques au titre de l'enseignement supérieur (2008)

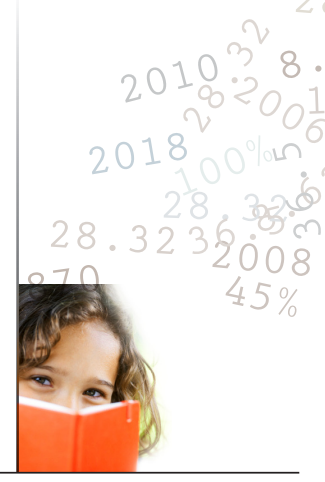

Ce graphique fait état des aides publiques octroyées aux ménages et aux autres entités privées au titre de l'éducation, en pourcentage des dépenses publiques totales d'éducation, ventilées par type d'aide.

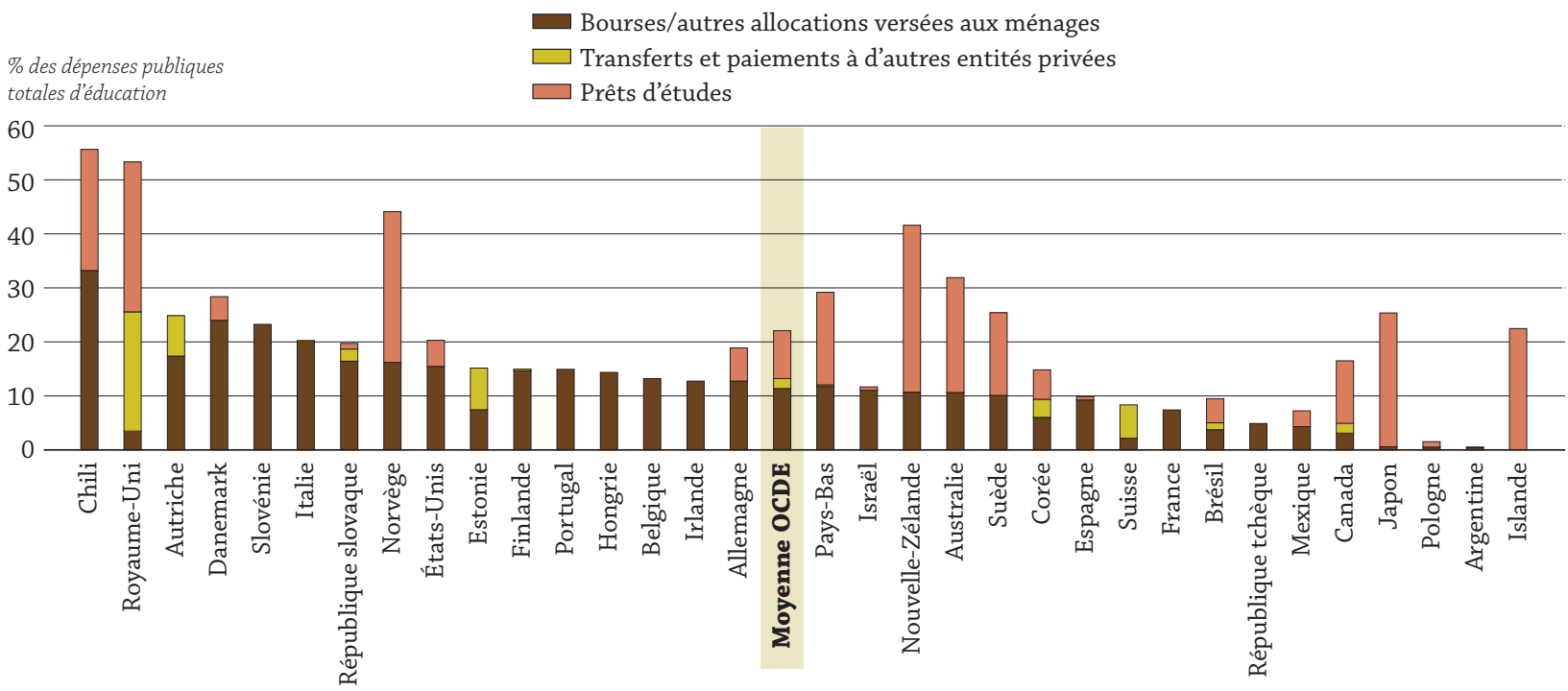

Source : Regards sur l'éducation 2011 : Les indicateurs de l'OCDE, Indicateur B5 (www.oecd.org/edu/eag2011). 


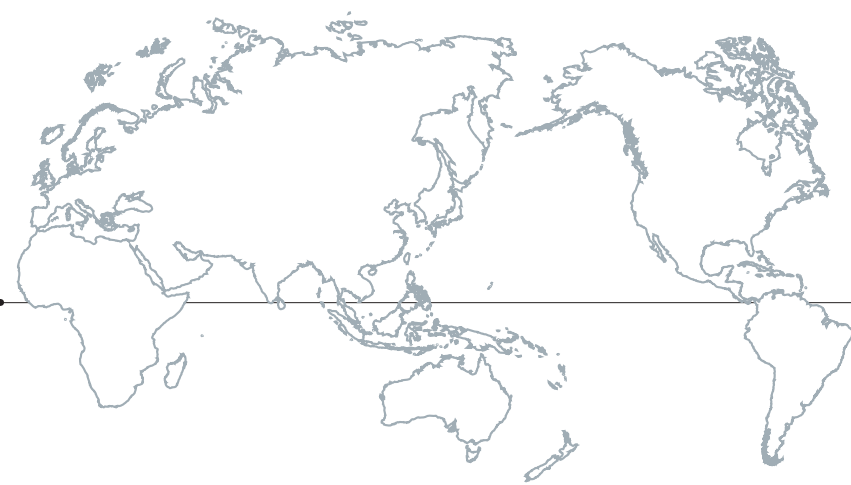

Les études de I'OCDE laissent penser que les systèmes d'aide financière aux étudiants qui allient à la fois prêts avec remboursement subordonné au revenu et allocations sous condition de ressources renforcent non seulement en amont l'accès et l'équité dans l'enseignement supérieur, mais permettent également en aval d'améliorer la réussite des étudiants. À titre d'exemple, l'Australie et la Nouvelle-Zélande ont utilisé cette approche pour atténuer l'impact de leurs frais de scolarité élevés, encourager l'accès à l'enseignement supérieur des élèves issus de milieux défavorisés et réduire le risque d'endettement lié aux prêts d'études. Parmi les autres pays de l'OCDE qui proposent des allocations sous condition de ressources et des prêts avec remboursement subordonné au revenu, citons le Chili, les États-Unis, les Pays-Bas et le Royaume-Uni, même si dans certains de ces pays, il est du ressort de l'emprunteur de faire la demande d'un remboursement subordonné au revenu.

\section{Il devient de plus en plus important de trouver un juste équilibre entre} financement public et financement privé.

Les pays se tournent également de plus en plus vers d'autres approches pour trouver le juste équilibre entre le maintien à un niveau raisonnable des frais à charge des étudiants et l'accès à des sources de financement suffisantes pour leurs systèmes d'enseignement supérieur. Ainsi, certains pays dont le système d'enseignement supérieur est fortement subventionné, tels que le Danemark et la Suède, ont augmenté ces dernières années les frais de scolarité à charge des étudiants non ressortissants de l'Union européenne, rejoignant la longue liste des pays qui appliquent des frais de scolarité plus élevés pour les étudiants en mobilité internationale. Dans au moins 14 pays membres ou partenaires de I'OCDE, les frais de scolarité varient en fonction du domaine d'études pour pallier le coût plus élevé de certains programmes. L'Australie a même tenté de lier le montant des frais de scolarité aux débouchés sur le marché du travail en abaissant les frais de scolarité dans les domaines où il existe une pénurie de compétences sur le marché du travail, afin de rendre ces filières plus attractives aux yeux des étudiants.

À mesure que 2012 avance, d'autres changements se font jour. Cette année, certaines universités anglaises ont prévu de tripler le montant de leurs frais de scolarité dans le cadre d'un plan gouvernemental destiné à stabiliser le financement des universités, et aux États-Unis, certains décideurs ont lancé un projet visant à lier l'éligibilité des étudiants aux aides financières au maintien des frais de scolarité à un niveau raisonnable par les établissements d'enseignement. En ces temps d'essor des effectifs scolarisés et de restrictions budgétaires, d'autres changements sont à prévoir, à n'en point douter.

Pour conclure Les systèmes de financement de l'enseignement supérieur qui pratiquent des frais de scolarité modérés - combinés à des systèmes d'aide financière proposant aux étudiants des prêts avec remboursement subordonné au revenu et des allocations sous condition de ressources - ont, selon toute vraisemblance, de meilleures chances de renforcer l'accès, l'équité et la réussite dans l'enseignement supérieur, tout en favorisant le succès des étudiants.

\begin{tabular}{l|l}
$\begin{array}{l}\text { Voir : } \\
\text { www.oecd.org/edu }\end{array}$ & $\begin{array}{l}\text { Consulter : } \\
\text { Regards surléducation 2011: } \\
\end{array}$ \\
& Les indicateurs de l'OCDE
\end{tabular}

\section{Pour plus d'informations, contacter :} J.D. LaRock (Jean-Daniel.LaRock@oecd.org)
Prochain numéro :

Comment les filles réussissent-elles à l'école et les femmes au travail - dans le monde? 